\title{
Massive pulmonary artery thrombosis with haemoptysis in adults with Eisenmenger's syndrome: a clinical dilemma
}

\author{
C Broberg, M Ujita, S Babu-Narayan, M Rubens, S K Prasad, J S R Gibbs, M A Gatzoulis
}

Heart 2004;90:e63 (http://www.heartinl.com/cgi/content/full/90/11/e63). doi: 10.1136/hrt.2004.039198

\begin{abstract}
Although the frequency of haemoptysis in Eisenmenger's syndrome is well recognised, the high prevalence of pulmonary artery thrombus has been newly appreciated through the growing use of non-invasive imaging. Three patients with Eisenmenger's syndrome with haemoptysis are reported who underwent computed tomography pulmonary angiography and cardiovascular magnetic resonance. Each patient was found to have aneurysmal dilatation of the right pulmonary artery with large laminar thrombus. These cases illustrate a rising clinical problem in this special populationthat is, how to treat and prevent large pulmonary artery thrombosis in the setting of haemoptysis. The authors discuss their approach to these cases and the known literature.
\end{abstract}

$\mathrm{F}$ or decades clinicians have appreciated the high frequency of haemoptysis in patients with Eisenmenger's syndrome. Wood's classic description of 127 patients reported haemoptysis in all of them by the age of 40 years. Haemoptysis does not predict prognosis ${ }^{2}$ and management of the problem in these patients has remained conservative. However, if large volume haemoptysis occurs the event may be fatal and clinicians have very little to offer. Wood ${ }^{1}$ reported that haemoptysis was the cause of death of $29 \%$ of 42 patients, and Daliento and colleagues ${ }^{2}$ reported that $11.4 \%$ of their series of fatal cases were caused by haemoptysis. The cause of haemoptysis is generally believed to be pulmonary infarction secondary to thrombosis as shown by many necropsy series. ${ }^{3}$ Even so, the precise site of bleeding cannot always be located at necropsy.

More recently, increased use of non-invasive imaging has shown massive underlying thrombi in a quarter of this patient group. Perloff and colleagues ${ }^{4}$ performed computed tomography (CT) pulmonary angiography (CTPA) in 31 patients, $29 \%$ of whom had large to massive thrombi. Silversides and colleagues ${ }^{5}$ published a similar series of 34 patients and found thrombus in $21 \%$. Female sex and low oxygen saturation were associated with higher incidence but there was no difference in New York Heart Association class, body mass index, haemoglobin, mean corpuscular volume, or platelet count. Other risk factors for thrombus have not been identified. The utility of cardiovascular magnetic resonance (CMR) in identification of these lesions has not been reported.

The coexistence of these two pathologically related problems, haemoptysis and pulmonary thrombosis, poses a difficult clinical dilemma. Although anticoagulation seems logical for both prevention and treatment of thrombosis, its use is far more complex in the setting of significant haemoptysis, and data on this issue are lacking. We report three recent cases of large pulmonary artery thrombus that illustrate this difficult dilemma.

\section{CASE SERIES \\ Case 1}

This 21 year old man with Down's syndrome has a partial atrioventricular septal defect and a non-restrictive patent ductus arteriosus measuring $1.0 \mathrm{~cm}$. By echocardiography the peak regurgitant velocity of his right atrioventricular valvethat is, the tricuspid valve-was $5.2 \mathrm{~m} / \mathrm{s}$. Basal resting oxygen saturation was $83 \%$ without differential cyanosis, and his haemoglobin was $237 \mathrm{~g} / \mathrm{l}$. He had maintained right and left ventricular systolic function. He was well until he suddenly expectorated approximately $250 \mathrm{ml}$ of bright red blood. CTPA was performed showing dramatic enlargement of the right pulmonary artery $(7.1 \mathrm{~cm})$, the majority of which was lined with calcified thrombus. CMR was also performed for anatomical clarification and to determine the existence of systemic-pulmonary collateral arteries (bronchial or intercostal). None were found. Since then, he has had six further episodes of large volume haemoptysis. On several of these he lost consciousness momentarily. Bleeding always stopped spontaneously after observation in hospital. Over six months his haemoglobin fell to $182 \mathrm{~g} / \mathrm{l}$ and he developed iron deficiency (transferrin saturation of $10 \%$ ). He has a normal platelet count. He has not taken warfarin.

\section{Case 2}

This 59 year old man has a large ventricular septal defect (VSD) measuring $2.0 \mathrm{~cm}$ and biventricular systolic dysfunction. Peak tricuspid valve velocity was $4.4 \mathrm{~m} / \mathrm{s}$. Oxygen saturation was $81 \%$ on air and haemoglobin was $204 \mathrm{~g} / \mathrm{l}$. He had experienced several episodes of small volume haemoptysis in the past. He developed a productive cough with abnormal findings on chest radiography, which led to him undergoing CTPA as an outpatient. The study showed pulmonary artery dilatation $(5.3 \mathrm{~cm})$ and a near circumferential mural thrombus in the right pulmonary artery with evidence of calcification within the thrombus. Warfarin was instigated. The patient returned five months later with large volume haemoptysis. His international normalised ratio was 2.3 , and his haemoglobin eventually dropped to as low as $179 \mathrm{~g} / \mathrm{l}$. CMR was performed to better define ventricular function. Despite five months of treatment, CMR still showed extensive thrombus. After cessation of warfarin he has had no further haemoptysis and his haemoglobin climbed gradually to $214 \mathrm{~g} / \mathrm{l}$.

\section{Case 3}

This 24 year old man has transposition of the great arteries and a non-restrictive VSD. At the age of 7 years he underwent a palliative Mustard procedure (atrial switch) without closure of the VSD (measuring $2.2 \mathrm{~cm}$ ). His peak tricuspid valve velocity was $4.6 \mathrm{~m} / \mathrm{s}$. He had several episodes of mild

Abbreviations: $C M R$, cardiovascular magnetic resonance; $C T$, computed tomography; CTPA, computed tomography pulmonary angiography; VSD, ventricular septal defect 
haemoptysis in the two years before presentation. He smoked cigarettes occasionally. He was recently hospitalised for worsening breathlessness, fatigue, and fevers. Resting heart rate was 90 beats/min with an oxygen saturation of $78 \%$. He had a haemoglobin concentration of only $109 \mathrm{~g} / \mathrm{l}$ with iron deficiency (serum iron of $4.0 \mu \mathrm{mol} / \mathrm{l}$ and transferrin saturation of $8 \%$ ). Blood cultures were repeatedly negative but $\mathrm{C}$ reactive protein was raised at $81 \mathrm{mg} / \mathrm{l}$ and he was treated empirically for endocarditis. CMR was performed to evaluate the atrial baffles and showed pulmonary artery dilatation $(10.3 \mathrm{~cm})$ and thrombus. CTPA was done to confirm these findings and define the extent of thrombus. This confirmed the aneurysmal pulmonary artery and mural thrombus, mainly on the superior aspect of the main and right pulmonary arteries. Warfarin and iron administration was started.

In each case CTPA was performed on a multidetector scanner (Somatom Volume Zoom; Siemens, Erlangen, Germany). Ninety millilitres of contrast media (Ultravist; Schering) was injected through a peripheral venous catheter with an automated injector with a flow of $4 \mathrm{ml} / \mathrm{s}$. Built-in software for bolus tracking (Carebolus; Siemens) was used to determine when CT density within the main pulmonary artery reached $100 \mathrm{HU}$ to signal the start of scanning. CT images were reconstructed with $1.25 \mathrm{~mm}$ sections at $1 \mathrm{~mm}$ intervals with a soft kernel. Transaxial images as well as oblique coronal reformation with maximum intensity projection are shown (fig l) and show striking aneurysmal enlargement of the right pulmonary artery with massive mural thrombus in each case. CMR was done with a $1.5 \mathrm{~T}$ system (Sonata; Siemens) with a phased array body coil. Anatomical and functional information was obtained from a combination of spin-echo and gradient-echo sequences. Phase velocity mapping provided information on flow patterns. Selected images of anatomy and thrombi are shown (fig 2).

\section{DISCUSSION}

We report three patients with Eisenmenger's syndrome with haemoptysis and remarkable dilatation of the pulmonary artery, more so on the right than the left, with large laminar thrombus. The location of these thrombi highly suggests that they originated in situ rather than embolically. Multiple calcifications within the thrombus indicate chronicity. Collateral arteries were not seen in any case, nor were clear areas of pulmonary infarction. All patients had severe cyanosis with secondary erythrocytosis (except in case 3 where iron deficiency was present) in the setting of pulmonary hypertension, and reflect the wide range of the clinical spectrum of Eisenmenger's syndrome.

Although haemoptysis occurs in the majority of patients with Eisenmenger's syndrome, with or without evidence of pulmonary thrombus, its presence makes treatment of these mural thrombi much more challenging. Data on whether anticoagulation is beneficial in either prevention or treatment are lacking, a topic that clearly needs to be addressed in clinical trials. Some have counselled against the routine use of anticoagulants or aspirin, ${ }^{6}$ whereas others have recommended urgent hospitalisation and heparinisation when thrombus is identified. ${ }^{7}$ Our own approach, admittedly empirical, is to encourage non-urgent outpatient warfarinisation in patients with identified thrombus and in whom haemoptysis is mild or absent. International normalised ratio should be measured with citrate adjusted tubes when packed cell volume is greater than $60 \%{ }^{8}$ If and when significant haemoptysis occurs, warfarin must be stopped until the patient's condition has stabilised. Iron storage should be measured and treated if low.
Case 1
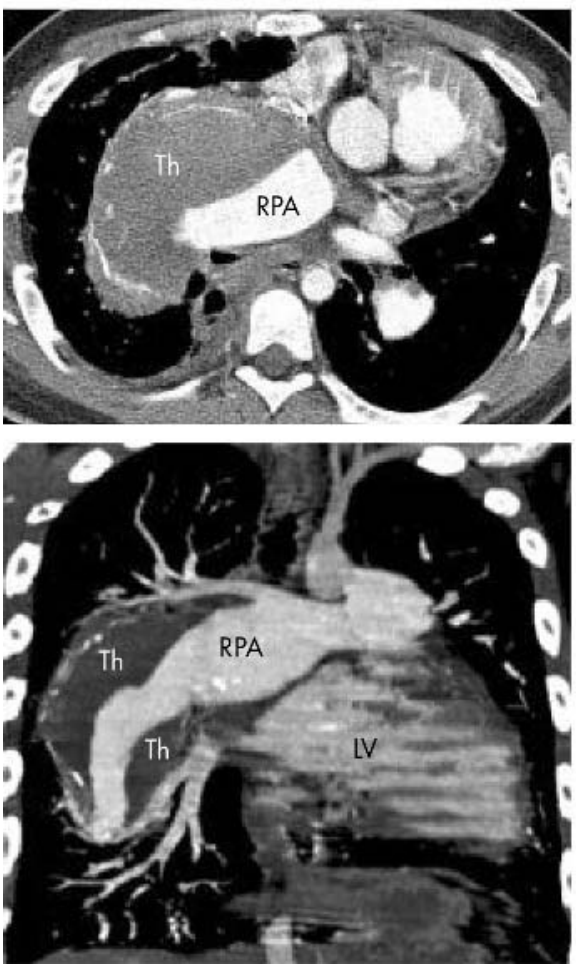

Case 2
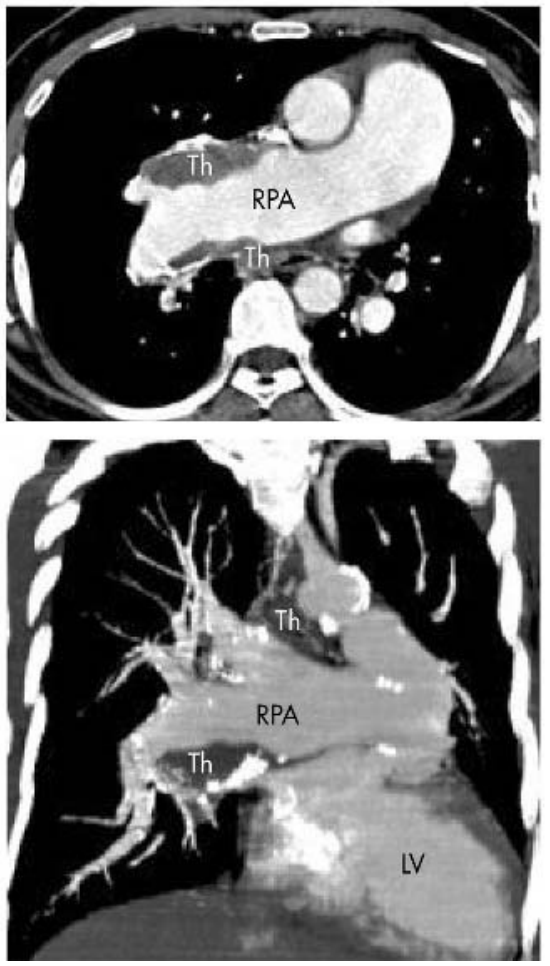

Case 3
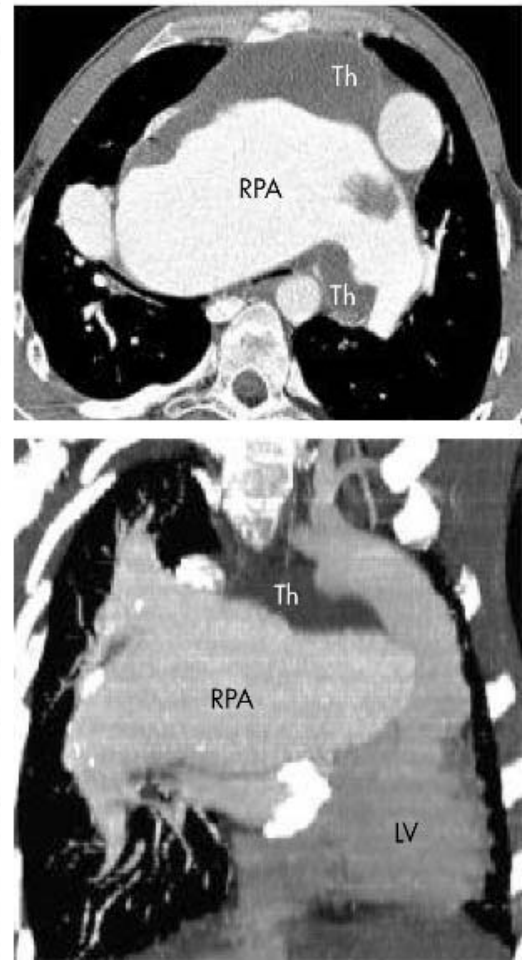

Figure 1 (Top) Computed tomography pulmonary angiography (CTPA) transaxial images and (bottom) oblique coronal reformation with maximum intensity projection for each patient. LV, left ventricle; RPA, right pulmonary artery; Th, thrombus. 
Case 1
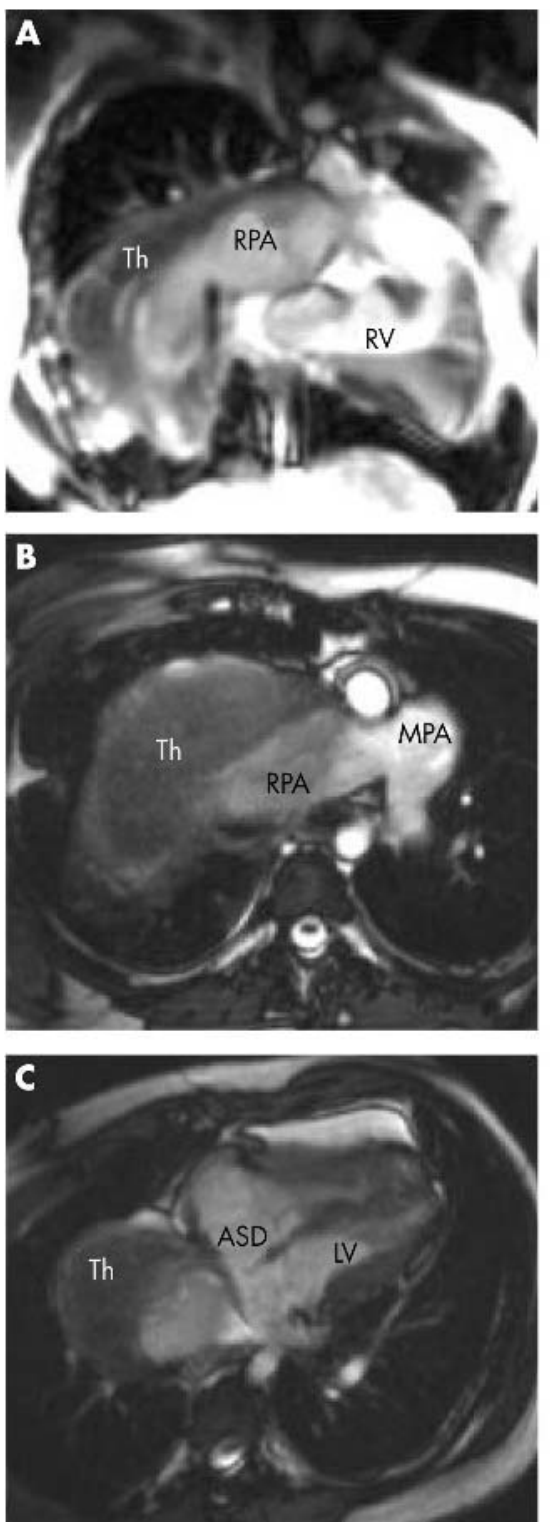

Case 2
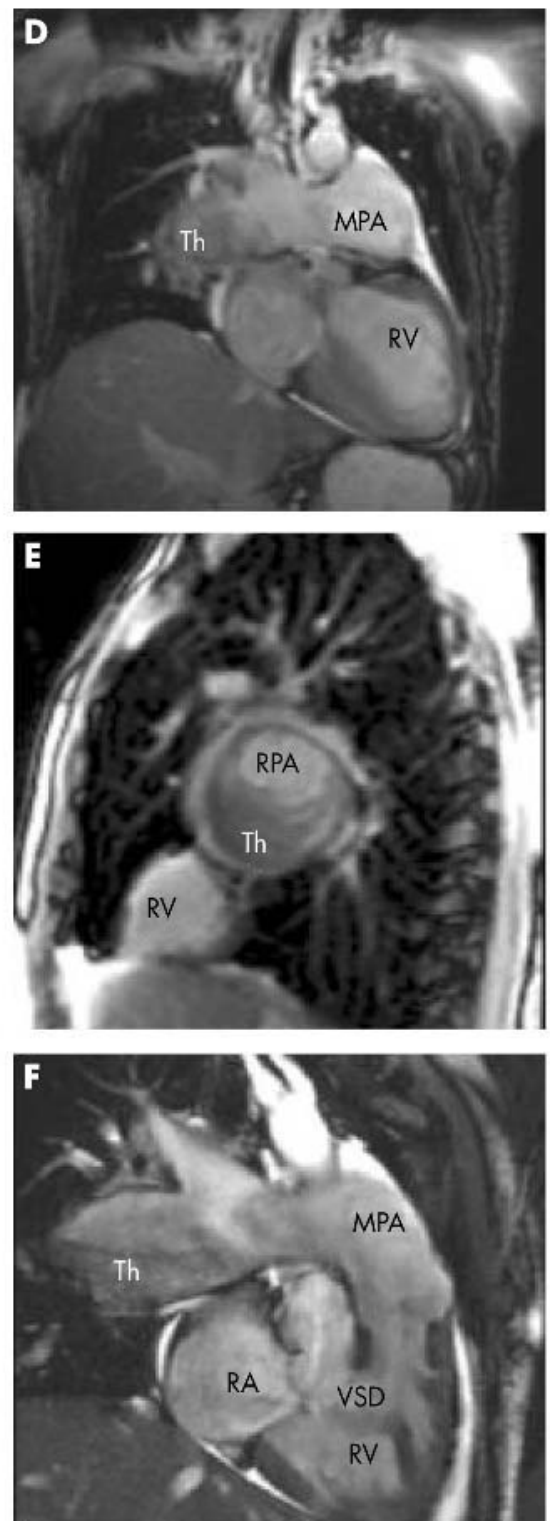

Case 3
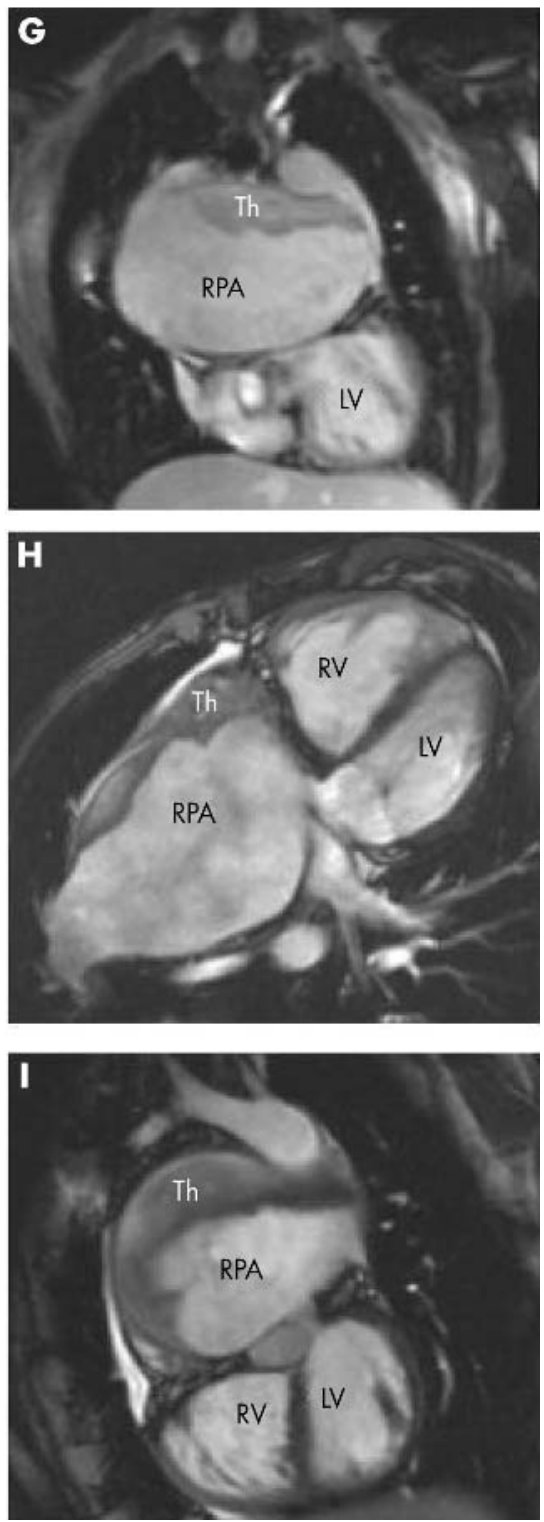

Figure 2 True fast imaging steady precession magnetic resonance images of each patient. ASD, atrial septal defect; MPA, main pulmonary artery; RA, right atrium; RV, right ventricle; Th, thrombus; VSD, ventricular septal defect. Case 1: (A) coronal slice showing the large RPA with Th;(B) transaxial slice showing the bifurcation of the MPA, with the RPA almost completely full of Th; (C) oblique transaxial cut in early systole showing the ASD. Case 2: (D) coronal slice; (E) sagittal slice through right lung, with Th occluding the majority of the RPA lumen; (F) oblique coronal slice showing the VSD. Case 3: (G) coronal slice with Th on the roof of the large RPA, with atrial baffles partly seen adjacent to the LV; (H) oblique transaxial slice showing anterior RV (systemic ventricle), posterior LV (pulmonary ventricle), and huge RPA with clot also anterior; (I) sagittal view from the left, showing the relation of LV to RPA.

This complex dilemma seems unique to the Eisenmenger group, where haemoptysis and thrombi are both common. In contrast, haemoptysis occurs in only $4 \%$ of patients with primary pulmonary hypertension and $3 \%$ of patients with uncomplicated atrial septal defect. ${ }^{1}$ In the series of Perloff and colleagues ${ }^{4}$ no thrombus was detected in the noncyanotic primary pulmonary hypertension group, even though $23 \%$ had calcification in the pulmonary arteries. Haemoptysis and thrombus must therefore be a product of both cyanosis and pulmonary vascular disease. Cyanosis has been linked to a number of abnormalities in clotting factors, which may contribute to thrombophilia, ${ }^{6}$ but the specific role of any of these in pulmonary thrombosis is unknown. The presence of bronchial artery hypertrophy may also be linked to haemoptysis and deserves further attention. ${ }^{9}$ It should be emphasised that haemoptysis and thrombus do not always coexist. Our own institution has a seen a number of cases of significant haemoptysis without thrombus, and vice versa, emphasising the complex underlying pathophysiological interplay.

Regardless of the presence of thrombi, large aneurysms of the pulmonary artery can themselves cause problems. Their large volume can cause displacement or compression of surrounding lung tissue, superior vena cava, or left recurrent laryngeal nerve. In addition, aneurysmal dissection or rupture has been reported, usually with fatal outcome. ${ }^{10}{ }^{11}$ In one recent case report, pulmonary artery dissection was successfully treated with urgent heart-lung transplantation. ${ }^{12}$ Otherwise surgical options have very limited value. Thrombectomy with aneurysm repair requiring bypass would 
be an extremely high risk operation, and pneumonectomy would not be sustainable given that patients with Eisenmenger's syndrome already have compromised pulmonary blood flow. There is perhaps a role for coil embolisation of systemic to pulmonary collateral vessels if present.

The high prevalence and severity of pulmonary artery thrombosis support the routine use of CTPA in patients with Eisenmenger's syndrome, even if they are asymptomatic. ${ }^{45}$ The role of CMR in thrombus detection has not been studied. Although in each of our cases these dramatic thrombi were easily seen, and in one case were first shown by MRI, the sensitivity of MRI compared with CTPA needs to be addressed. The real advantage of MRI is in better definition of cardiac anatomy and function and avoidance of radiation exposure. Other modalities such as ventilation-perfusion scans or transcatheter biplane pulmonary angiography look for lumen obstruction and thus may in theory miss laminar clots such as those shown here.

The clinical management of pulmonary artery thrombus and haemoptysis is an important and difficult problem. Further work is warranted incorporating the better imaging modalities now available.

\section{Authors' affiliations}

C Broberg, S Babu-Narayan, S K Prasad, J S R Gibbs, M A Gatzoulis, Division of Cardiology, Royal Brompton Hospital and Harefield NHS

Trust, London, UK

M Ujita, M Rubens, Department of Radiology, Royal Brompton Hospital and Harefield NHS Trust
Correspondence to: Dr Michael A Gatzoulis, Adult Congenital Heart Disease Unit, Royal Brompton Hospital, Sydney Street, London SW3 6NP, UK; m.gatzoulis@rbh.nthames.nhs.uk

Accepted 13 July 2004

\section{REFERENCES}

1 Wood $\mathbf{P}$. The Eisenmenger syndrome or pulmonary hypertension with reversed central shunt. BMJ 1958;ii:701-9, 755-62.

2 Daliento L, Somerville J, Presbitero P, et al. Eisenmenger syndrome: factors relating to deterioration and death. Eur Heart J 1998;19:1845-55.

3 Canada WJ, Goodale F Jr, Currens JH. Defect of the interatrial septum, with thrombosis of the pulmonary artery; report of three cases. N Engl J Med 1953;248:309-16.

4 Perloff JK, Hart EM, Greaves SM, et al. Proximal pulmonary arterial and intrapulmonary radiologic features of Eisenmenger syndrome and primary pulmonary hypertension. Am J Cardiol 2003;92:182-7.

5 Silversides CK, Granton JT, Konen E, et al. Pulmonary thrombosis in adults with Eisenmenger syndrome. J Am Coll Cardiol 2003;42:1982-7.

6 Perloff JK, Rosove MH, Child JS, et al. Adults with cyanotic congenital heart disease: hematologic management. Ann Intern Med 1988;109:406-13.

7 Somerville J. How to manage the Eisenmenger syndrome. Int J Cardiol 1998;63:1-8.

8 Oechslin E. Eisenmenger's syndrome. In: Gatzoulis MA, Webb GD, Daubeney $P$, eds. Diagnosis and management of adult congenital heart disease. Philadelphia: Churchill Livingstone, 2003:363-77.

9 Deffebach ME, Charan NB, Lakshminarayan S, et al. The bronchial circulation: small, but a vital attribute of the lung. Am Rev Respir Dis 1987; 135:463-81.

10 Placik B, Rodbard S, McMahon J, et al. Pulmonary artery dissection and rupture in Eisenmenger's syndrome. Vasc Surg 1976;10:72-80.

11 Areco D, Pizzano N. Pulmonary artery dissection: echocardiographic findings and diagnosis. Echocardiography 2003;20:375-7.

12 Tonder N, Kober L, Hassager C. Pulmonary artery dissection in a patient with Eisenmenger syndrome treated with heart and lung transplantation. Eur J Echocardiogr 2004;5:228-30. 